\title{
Nanoimprinted Semitransparent Metal Electrodes and Their Application in Organic Light-Emitting Diodes**
}

\author{
By Myung-Gyu Kang and L. Jay Guo*
}

Organic light-emitting diodes (OLEDs) are promising for full-color, full-motion, flat panel display applications ${ }^{[1-3]}$ because they offer several advantageous features, for example, ease of fabrication, low costs, light weight, bright self-emission, a wide viewing angle, and the possibility of flexible displays. The basic OLED structure consists of a number of organic semiconductor layers sandwiched between a cathode and an anode. For efficient electron injection into the organic layers, low-work-function materials are required for the cathode. A very thin $\mathrm{LiF}$ layer with a thick $\mathrm{Al}$ capping is widely used for this purpose. ${ }^{[4]}$ For the anode, indium tin oxide (ITO) is the predominant choice because it offers transparency in the visible range of the electromagnetic spectrum as well as electrical conductivity. ${ }^{[5-7]}$

However, several aspects of ITO are far from optimal for high-performance OLEDs. It is known that the migration of indium and oxygen from ITO into organic semiconductors during OLED operation causes device degradation. ${ }^{[8,9]}$ The electrical properties of ITO greatly depend on the film preparation. ${ }^{[10,11]}$ The rough surface of the deposited ITO film and the work function of ITO, ca. $4.7 \mathrm{eV}$, limit the efficiency of the hole injection. ${ }^{[12]}$ The typical sheet resistance of a $100 \mathrm{~nm}$ thick ITO layer, $20-80 \Omega / \square$, is still high, which causes a voltage drop along the addressing line, thus limiting the operation of a large-area passive matrix OLED array. ${ }^{[13]}$ Moreover, the cost of ITO has escalated in recent years because of the jump in price of the element indium. Several alternative materials, for example, TiN, ${ }^{[14]} \mathrm{Al}$-doped $\mathrm{ZnO},{ }^{[15]}$ and fluorine tin oxide, ${ }^{[16]}$ have been investigated as anode materials instead of ITO; however, none are optimal as anode in OLEDs because they have either a lower work function or a lower conductivity than ITO. Other transparent conducting oxides, such as Ga-In-Sn-O (GITO), Zn-In-Sn-O (ZITO), Ga-In-O (GIO), and $\mathrm{Zn}-\mathrm{In}-\mathrm{O}$ (ZIO), that have a higher work function and a similar electrical conductivity when compared to ITO have also been examined as OLED anode materials. ${ }^{[17]}$ However, they are potentially problematic because they also contain the element indium that i) may diffuse into the organic

[*] Prof. L. J. Guo, M.-G. Kang

Department of Electrical Engineering and Computer Science The University of Michigan

1301 Beal Ave., Ann Arbor, MI 48109-2122 (USA)

E-mail: guo@eecs.umich.edu

[***] This work was supported in part by NSF grant ECS 0424204 . layer in the OLED; and ii) has a high price, making these electrodes expensive. Besides these materials several metals with a high work function, such as $\mathrm{Au},{ }^{[12]} \mathrm{Ni}^{[18]}$ and $\mathrm{Pt},{ }^{[19]}$ have been investigated as anodes for OLEDs. In these cases the metal was used to modify the surface of the ITO electrode, or as an anode for top-emitting devices. A surface-modified thin $\mathrm{Ag}$ film ${ }^{[20,21]}$ has been used as a semitransparent electrode instead of ITO, but its transparency was low. Recently, carbon nanotube films have been investigated as transparent, conductive electrodes, ${ }^{[22-25]}$ but they have a high sheet resistance that may limit the device performance.

In this Communication, we report semitransparent metal electrodes fabricated by nanoimprint lithography (NIL), and evaluate their potential as OLED anodes. NIL, an emerging lithographic technique, ${ }^{[26,27]}$ is well-suited to the area of organic electronics, which requires low-cost and high-throughput fabrication at high resolution. The fabricated semitransparent metal electrode offers several advantages over ITO for OLED applications. First, several problems associated with ITO can be eliminated, such as device degradation by indium diffusion and high costs. Second, efficient hole injection into the organic semiconductor can be realized by choosing metals with a high work function, such as $\mathrm{Au}$ or Pt. Third, a semitransparent metal electrode is potentially suitable for topemitting devices and tandem structures. Last, but not least, the output efficiency of the OLED can be enhanced by preventing waveguiding in the ITO layer, which occurs as a result of its high refractive index and is one of the limitations to the external efficiency of OLEDs, ${ }^{[28]}$ and by forming a two-dimensional (2D) hole array with proper periodicity. ${ }^{[29,30]}$ We demonstrate here that a unique property of such an electrode is that its optical transparency and the electrical conductivity can be tuned separately by changing the aperture ratio and the metal thickness, thereby making it possible to tailor the structures for different applications. To our knowledge, a nanoimprinted semitransparent metal electrode has not been reported before.

The semitransparent metal electrodes are in the form of a nanometer-scale periodically perforated dense metal mesh on glass. Two design considerations led to such structures: i) the line width of the metal mesh was designed to be subwavelength, to provide sufficient transparency and to minimize light scattering; and ii) the period of the mesh was chosen to be sub-micrometer to ensure the uniformity of the current injection into the organic semiconductors. Such large-area dense nanostructures can be fabricated by NIL, which is ideal for this application because of its inherently high resolution 
and its high-throughput features. Metal mesh structures have been fabricated by NIL by using a mold with periodic grid patterns, followed by a standard metal deposition and lift-off process. The rectangular grid pattern on the imprinting mold (Fig. 2a) is created by applying NIL twice, using two grating molds. One grating mold has a period of $700 \mathrm{~nm}$, which defines the main part of the semitransparent metal electrode, while the second grating mold, imprinted orthogonally to the $700 \mathrm{~nm}$ period grating, has a period of $10 \mu \mathrm{m}$ and is used to ensure the electrical connectivity of the $700 \mathrm{~nm}$ period grating lines, in case some lines are disconnected because of defects in the fabrication processes. The horizontal metal lines produced by the second grating also help to distribute the current flow in the semitransparent electrodes in a quasi-2D fashion, which is important for addressing the OLED devices in certain applications.

Optical transparency and electrical conductivity are the two most important factors for transparent electrodes. These factors can be quantified experimentally by measuring the transmittance and the sheet resistance, respectively. The parameters for tuning transmittance and sheet resistance in our structure are the mesh line width, the metal thickness, and the periodicity of both grating lines. The metal thickness and the overall mesh fill factor determine the sheet resistance of the structure. We will show that these two parameters can be controlled separately, providing sufficient design latitude for optimal performance. In the Experimental section we introduce a simple technique, based on imprinting and shadow evaporation, that allows grating structures with various line widths to be fabricated while keeping the same period. By using this technique, we were able fabricate large-area metal meshes of different line widths. To increase the transparency of the nanostructured metal electrode, a $700 \mathrm{~nm}$ period grating mold with narrow line width was first fabricated by shadow evaporation, as shown in Figure 1.

We will first demonstrate that the transmittance can be tuned by changing the opening ratio in the metal mesh structure. Two nanoimprint molds having the same period (700 nm) but with different duty cycles were fabricated by changing the metal thickness during shadow evaporation of $\mathrm{Cr}$, as shown in Figure 1. One mold has a $200 \mathrm{~nm}$ line width $(500 \mathrm{~nm}$ trench) while the other has a $120 \mathrm{~nm}$ line width (580 nm trench). A semitransparent metal electrode with high transmittance can be fabricated by using these molds. For example, $40 \mathrm{~nm}$ thick Au electrodes with line widths of 200 and $120 \mathrm{~nm}$ give an average transmittance of $69 \%$ and $81 \%$, respectively, while for a nonpatterned Au film with the same thickness the transmittance is only $6.7 \%$. After the fabrication of molds with orthogonal grating patterns (Fig. 2a and b), NIL was performed to transfer the rectangular patterns in the mold onto a resist layer on a glass substrate. Standard oxygen residual etching, metallization, and liftoff completed the fabrication of the semitransparent metal electrodes on glass. Figure $2 \mathrm{c}$ and d shows scanning electron microscopy (SEM) images of the fabricated metal electrodes on glass.
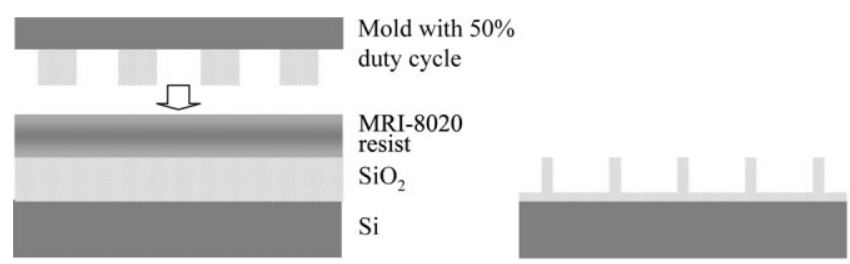

Nanoimprint

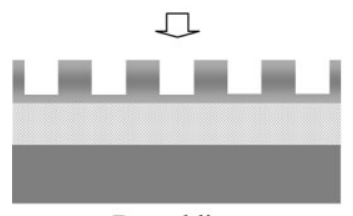

Demolding

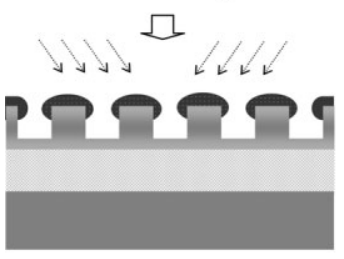

Shadow evaporation of $\mathrm{Cr}$
$\mathrm{SiO}_{2}$ etch and removal of $\mathrm{Cr}$ 七

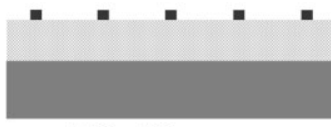

Lift-off in acetone

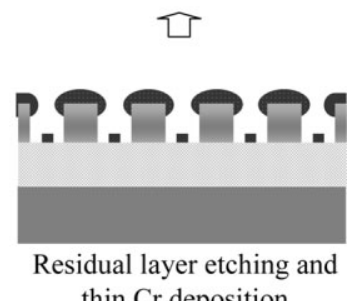

thin $\mathrm{Cr}$ deposition

Figure 1. Schematic illustration of the fabrication procedure of the narrow-line-width mold.

To characterize the optical transmittance and the sheet resistance $\left(R_{\square}\right)$ of the semitransparent electrodes, two sets of samples having large-area rectangular grids were fabricated. One had a line width of $200 \mathrm{~nm}$, a metal thickness $40 \mathrm{~nm}$, and a $2 \mathrm{~nm}$ Ti adhesion layer; while the other had a line width of $120 \mathrm{~nm}$, metal thicknesses of 40, 60, and $80 \mathrm{~nm}$, and a $2 \mathrm{~nm} \mathrm{Ti}$ adhesion layer. Several metals, such as $\mathrm{Au}, \mathrm{Cu}$, and $\mathrm{Al}$, were tested in the experiment. Figure 3 shows transmission spectra for each electrode structure.

For metal electrodes with a line width of $200 \mathrm{~nm}$, the average transmittance in the visible range is $63 \%, 58 \%$, and $61 \%$ for $\mathrm{Au}, \mathrm{Al}$, and $\mathrm{Cu}$ electrodes, respectively. As shown in Figure $3 \mathrm{a}$, the transmittance is strongly dependent on wavelength in these cases, and the trend of wavelength dependence is different for different metals. We can increase the transmittance by reducing the metal line width from $200 \mathrm{~nm}$ to $120 \mathrm{~nm}$, as shown in Figure 3b-d. The average transmittance is increased by more than $10 \%$ compared with electrodes with a line width of $200 \mathrm{~nm}$ but the same thickness. Moreover, the wavelength dependence is greatly reduced by using a smaller line width. The average transmittance for the metal electrodes with $120 \mathrm{~nm}$ line width and the same thickness $(40 \mathrm{~nm})$ is $74 \%, 75 \%$, and $73 \%$ for the $\mathrm{Au}, \mathrm{Al}$, and $\mathrm{Cu}$ electrodes, respectively. The transmittance in the longer-wavelength region (above $700 \mathrm{~nm}$ ) is over $80 \%$ for all three metals. At shorter wavelengths the behavior of the various metals differs. The $\mathrm{Au}$ electrode shows high transmittance in the short-wavelength region (below $500 \mathrm{~nm}$ ) and low transmittance in the medium-wavelength region (between $500 \mathrm{~nm}$ and $700 \mathrm{~nm}$ ). 

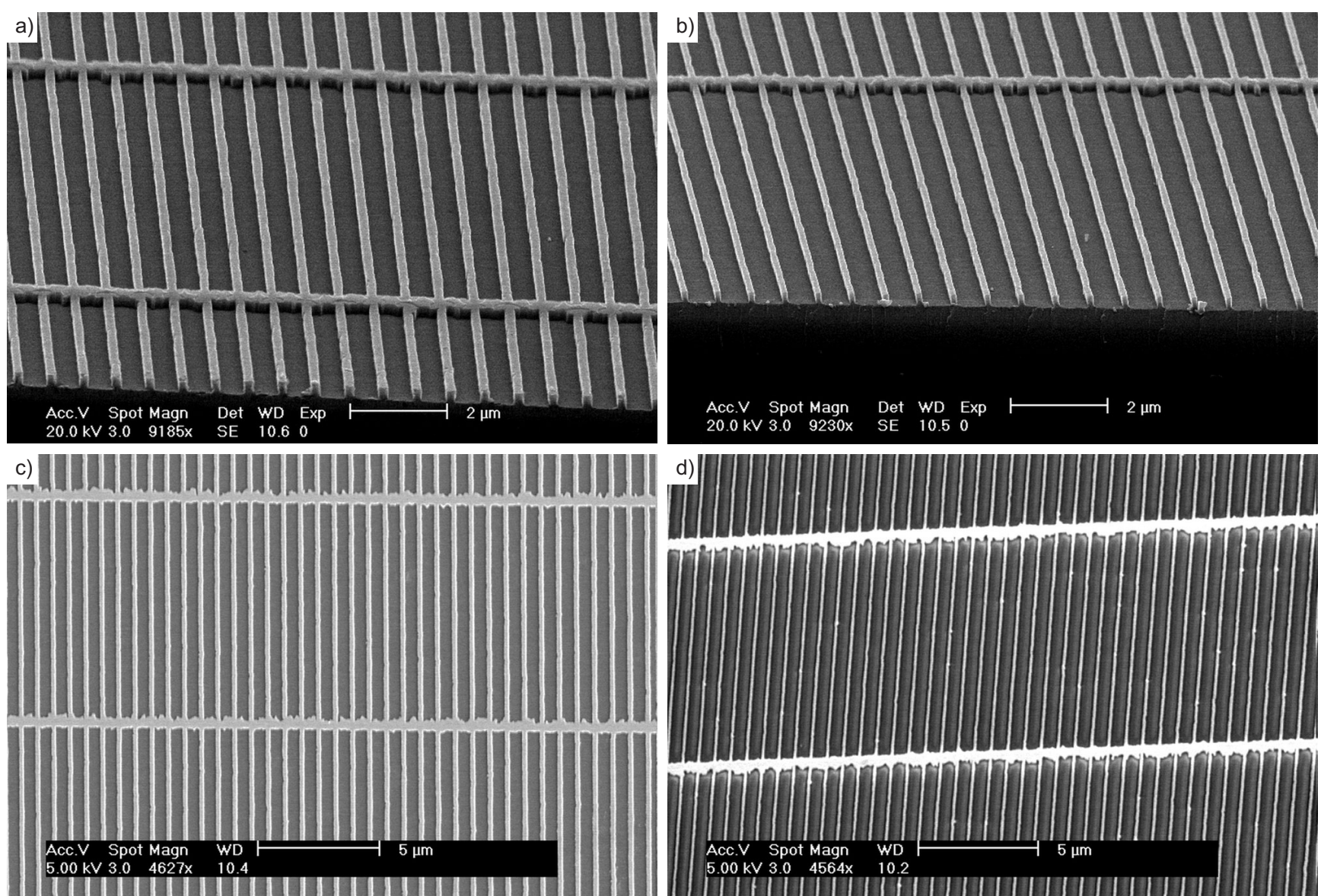

Figure 2. Scanning electron microscopy images of imprinting molds with rectangular grids of line widths of a) $200 \mathrm{~nm}$ and b) $120 \mathrm{~nm}$, and the corresponding semitransparent metal electrodes (on glass) with line widths of c) $200 \mathrm{~nm}$ and d) $120 \mathrm{~nm}$.

The Al electrode shows a flatter transmittance than the $\mathrm{Au}$ electrode, and the transmittance increases gradually as the wavelength increases. The $\mathrm{Cu}$ electrode shows an intermediate behavior compared with the $\mathrm{Au}$ and $\mathrm{Al}$ electrodes. The wavelength dependence of transmittance is correlated to the plasmon absorption band in the different metals.

The increase in transmittance achieved by reducing the metal line width is accompanied by an increase in sheet resistance, but such an increase of sheet resistance can be compensated for by using thicker metals. The average transmittance and sheet resistance for metal electrodes with a line width of $120 \mathrm{~nm}$ and several thicknesses are summarized in Figure 4. The sheet resistance for the metal electrodes with a line width of $200 \mathrm{~nm}$ and a thickness $40 \mathrm{~nm}$ is $7.68,8.45$, and $10.8 \Omega / \square$ for $\mathrm{Au}, \mathrm{Al}$, and $\mathrm{Cu}$ electrodes, respectively. For the $\mathrm{Au}$ electrode, the sheet resistance increases from 7.68 to $11.7 \Omega / \square$ when reducing the line width from $200 \mathrm{~nm}$ to $120 \mathrm{~nm}$ for higher transmittance. The increase in sheet resistance of the $\mathrm{Al}$ and $\mathrm{Cu}$ electrodes is similar to that of the $\mathrm{Au}$ electrode. As shown in Figure 4a, the sheet resistance can be significantly reduced by making the metal electrode thicker. For example, the sheet resistance of $11.7 \Omega / \square$ for the $40 \mathrm{~nm}$ thick Au electrode decreases to $4.79 \Omega / \square$ for the $80 \mathrm{~nm}$ thick Au electrode, with only a small reduction in transmittance. For all the considered cases the sheet resistance decreases by more than $59 \%$, but the transmittance decreases by less than $8 \%$ on increasing the thickness of the metal from $40 \mathrm{~nm}$ to $80 \mathrm{~nm}$. These results are plotted in Figure $4 \mathrm{~b}$. It can be seen from the fitted line that the sheet resistance could be further decreased to less than a few ohms per square with only a small decrease of average transmittance if metals thicker than $80 \mathrm{~nm}$ were to be used. These results confirm that the transmittance and sheet resistance of the semitransparent metal electrode can be easily tuned by varying the metal line width and thickness.

An important observation can be made from these results, namely that the transparency of such an electrode structure can be increased without sacrificing the overall conductivity by using narrower and thicker metal lines. This characteristic is another advantage over an ITO electrode, in which the sheet resistance has to be compromised to achieve high transmittance. Therefore, nanoimprinted semitransparent metal electrodes are very promising, not only because they can avoid the disadvantages of the ITO electrode but also because they can meet the requirements of both sheet resistance and optical transmittance when the duty cycle of the 



Figure 3. Transmittance of a) a metal electrode with a line width of $200 \mathrm{~nm}$ and b) $\mathrm{Au}, \mathrm{c}$ ) $\mathrm{Cu}$, and d) $\mathrm{Al}$ electrodes with a line width of $120 \mathrm{~nm}$.
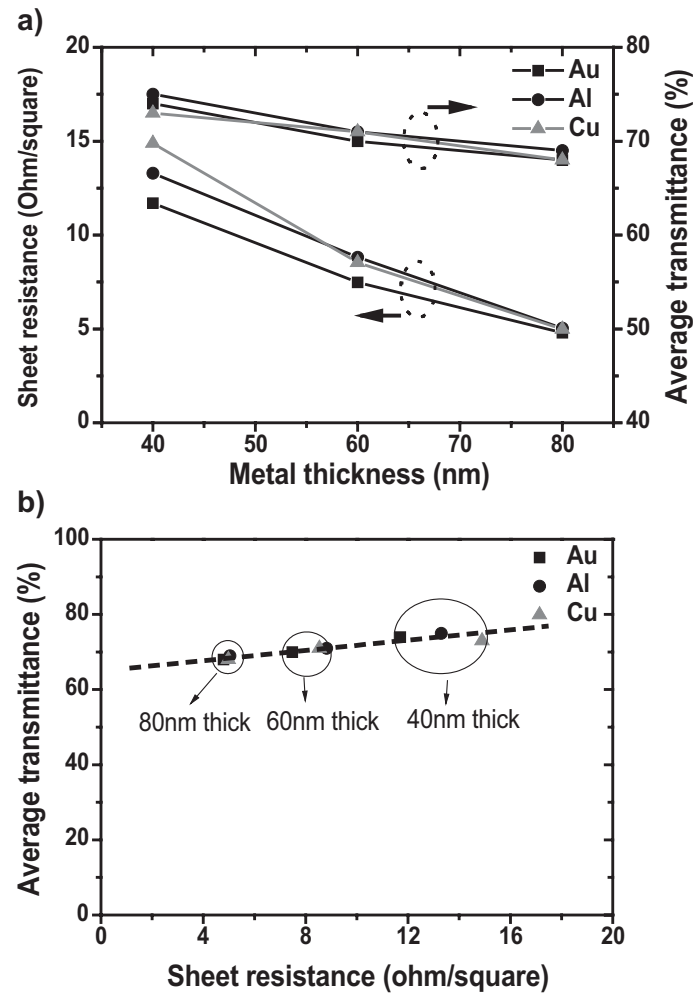

Figure 4. a) Sheet resistance and average transmittance as a function of metal thickness and b) Average transmittance vs. sheet resistance of semitransparent metal electrodes with a line width of $120 \mathrm{~nm}$. periodic patterns on the mold and the metal thickness are optimized.

To demonstrate the potential use of semitransparent metal electrodes as a transparent conducting electrode for optoelectronic devices, an OLED was fabricated using such an electrode as the anode. Although $\mathrm{Au}$ is preferred because of its high work function for efficient hole injection, we present here results obtained with $\mathrm{Cu}$ as the anode material because of its much lower costs. $\mathrm{Cu}$, being one of the cheapest metals, is well suited for practical organic electronic applications as it has a similar work function to ITO (ca. $4.7 \mathrm{eV}$ ). Semitransparent $\mathrm{Cu}$ electrodes with a thickness of $40 \mathrm{~nm}$ and line widths of 200 and $120 \mathrm{~nm}$ were chosen as anodes in the OLEDs. The OLED device consists of a semitransparent $\mathrm{Cu}$ mesh as the anode, poly(styrene sulfonate)-doped poly(3,4-etylenedioxythiophene) (PEDOT) as the hole transport layer, poly(2-(2-ethylhexyloxy)-5-methoxy-1,4-phenylenevinylene) (MEH-PPV) as the emissive layer, and $\mathrm{LiF} / \mathrm{Al}$ as the cathode. Device fabrication details can be found in the Experimental section.

Figure 5 shows current density versus voltage and electroluminescence characteristics of the OLEDs using semitransparent $\mathrm{Cu}$ anodes. The junction exhibits good rectifying characteristics. The turn-on voltages of these OLEDs are similar to the control samples made with ITO electrodes. The OLED made with the $120 \mathrm{~nm} \mathrm{Cu}$ anode shows a slightly higher turnon voltage than the device made with the $200 \mathrm{~nm} \mathrm{Cu}$ anode and, as a result, lower current density at a given bias after the device is turned on. As a comparison, the current density versus voltage characteristic of an OLED made with a $40 \mathrm{~nm}$ thick continuous $\mathrm{Cu}$ film as the anode is also shown in Figure 5a. It shows a similar characteristic to the OLED made with the nanoimprinted $\mathrm{Cu}$ anode, only with a slightly higher current density at a given bias. From these results, we can deduce that the overall mesh fill factor does not significantly affect the device's electrical performance. Both devices show strong electroluminescence peaked at $577 \mathrm{~nm}$, which is the same as the photoluminescence (PL) peak of MEH-PPV, as shown in Figure 5b. Although we do not have a setup to quantify the total output power of these OLEDs, to the naked eye the device made with the semitransparent $\mathrm{Cu}$ electrode is as bright as the control sample made using the ITO electrode. Upon optimization, such a semitransparent metal electrode could be used to replace the ITO anode.

In summary, we have introduced a method to fabricate a semitransparent metal electrode in the form of a nanometer- 

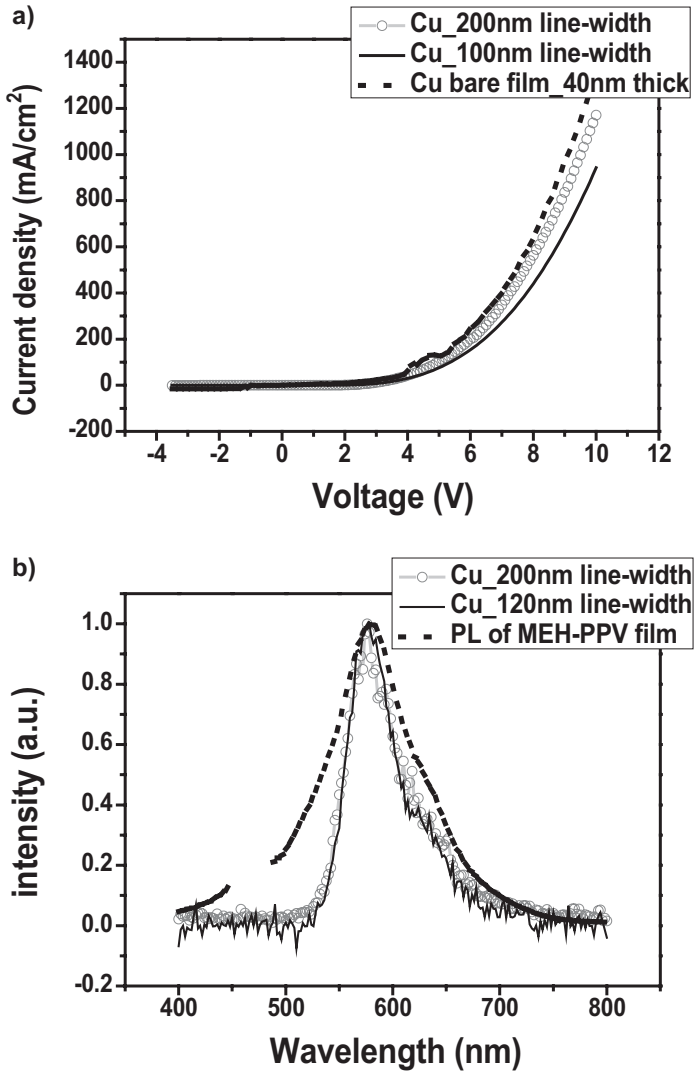

Figure 5. a) Current density vs. applied voltage and b) electroluminescence characteristics of OLEDs with a semitransparent $\mathrm{Cu}$ anode.

scale periodically perforated metal film that shows high optical transmittance in the visible range of the electromagnetic spectrum as well as excellent electrical conductivity. As an example application, an OLED was fabricated using a semitransparent $\mathrm{Cu}$ electrode as the anode to replace the conventional ITO electrode. A semitransparent metal electrode can be used instead of ITO in many other applications for which specific transmittance and conductivity are required, because they are easily tuned by varying the line width and the thickness of the metal. Moreover, the work function of an anode can be easily changed by choosing different metal materials, which provide more flexibility in studying the effect of the anode work function on device behavior. Furthermore, a similar structure can be made on a flexible plastic substrate, and will be the subject of a forthcoming publication. Our results indicate that semitransparent metal electrodes are an attractive and potentially practical solution for organic devices such as OLEDs and organic solar cells.

\section{Experimental}

Fabrication of the Narrow-Line-Width Mold: This was critical step as the degree of the transmittance mainly depends on the opening ratio of the fabricated electrode. A Nanonex NX2000 nanoimprinter (Princeton, NJ) was used for the NIL process. For the fabrication of the $700 \mathrm{~nm}$ period mold with a narrow line width an original $700 \mathrm{~nm}$ period $\mathrm{SiO}_{2}$ grating mold with a $50 \%$ duty cycle was used to imprint a MRI-8020 resist (Microresist Technology GmbH) spin-coated onto a $\mathrm{SiO}_{2}$ substrate at a pressure of $600 \mathrm{psi}(1 \mathrm{psi} \approx 6.89 \mathrm{kPa})$ and a temperature of $180^{\circ} \mathrm{C}$ for $5 \mathrm{~min}$. After the sample had been cooled and demolded, $\mathrm{Cr}$ was deposited via shadow evaporation by using electronbeam evaporation on each side of the imprinted resist, as shown in Figure 1 . The metal line width could be easily varied at this stage by changing the thickness of the evaporated $\mathrm{Cr}$. Anisotropic oxygen plasma etching was then used to remove the residual resist in the imprinted areas. Subsequently, an additional thin $\mathrm{Cr}$ layer was deposited and used as a $\mathrm{SiO}_{2}$ etching mask after liftoff in acetone. $\mathrm{SiO}_{2}$ was then etched using $\mathrm{CHF}_{3}$ at $20 \mathrm{sccm}$ for $10 \mathrm{~min}$, at a power of $150 \mathrm{~W}$ and a pressure of $20 \mathrm{mTorr}(1 \mathrm{mTorr} \approx 133 \mathrm{mPa})$, resulting in a grating of $200 \mathrm{~nm}$ depth. Treatment of the mold with an antistick layer ( $1 \mathrm{H}, 1 \mathrm{H}, 2 \mathrm{H}, 2 \mathrm{H}$-perfluorodecyl trichlorosilane) after Cr removal completed the fabrication of the mold with increased trench width.

OLED Fabrication: Semitransparent $\mathrm{Cu}$ anodes on glass were cleaned in acetone and isopropyl alcohol (IPA) under sonication for $20 \mathrm{~min}$ each and then exposed to oxygen plasma to thoroughly remove organic residue. The plasma treatment also made the metal anode more hydrophilic, allowing for easy spin-coating of the PEDOT hole-transport layer. Filtered PEDOT was spin-coated onto the $\mathrm{Cu}$ anode at $1500 \mathrm{rpm}$ for $30 \mathrm{~s}$, producing an $80 \mathrm{~nm}$ thick layer, and then baked at $120^{\circ} \mathrm{C}$ for $1 \mathrm{~h}$. Subsequently, the emissive organic layer MEH-PPV ( $0.5 \%$ by weight, dissolved in toluene) was spin-coated after filtration onto the PEDOT layer at $1000 \mathrm{rpm}$ for $30 \mathrm{~s}$, which gave a $50 \mathrm{~nm}$ thick layer, and then baked in a vacuum oven for $24 \mathrm{~h}$ at ambient temperature. Thermal evaporation of a $1 \mathrm{~nm}$ thick $\mathrm{LiF}$ layer followed by a $100 \mathrm{~nm}$ thick Al layer through a shadow mask completed the OLED device fabrication.

Received: January 17, 2007

Revised: February 17, 2007 Published online: April 25, 2007

[1] C. W. Tang, S. A. Van Slyke, Appl. Phys. Lett. 1987, 51, 913.

[2] J. H. Burroughes, D. D. C. Bradley, A. R. Brown, R. N. Marks, K. Mackay, R. H. Friend, P. L. Burn, A. B. Holmes, Nature 1990, $347,539$.

[3] P. E. Burrows, G. Gu, V. Bulovic, Z. Shen, S. R. Forrest, M. E. Thompson, IEEE Trans. Electron Devices 1997, 44, 1188.

[4] Y. D. Jin, X. B. Ding, J. Reynaert, V. I. Arkhipov, G. Borghs, P. L. Heremans, M. van der Auweraer, Org. Electron. 2004, 5, 271.

[5] A. D. H. H. J. Szczyrbowski, Phys. Status Solidi A 1983, 78, 243.

[6] K. Sreenivas, T. S. Rao, M. Abhai, C. Subhash, J. Appl. Phys. 1985, $57,384$.

[7] M. Higuchi, S. Uekusa, R. Nakano, K. Yokogawa, Jpn. J. Appl. Phys., Part 1 1994, 1, 302.

[8] J. R. Sheats, D. B. Roitman, Synth. Met. 1998, 95, 79.

[9] M. P. de Jong, D. P. L. Simons, M. A. Reijme, L. J. van IJzendoorn, A. W. Denier van der Gon, M. J. A. de Voigt, H. H. Brongersma, R. W. Gymer, Synth. Met. 2000, 110, 1.

[10] J. S. Kim, M. Granstrom, R. H. Friend, N. Johansson, W. R. Salaneck, R. Daik, W. J. Feast, F. Cacialli, J. Appl. Phys. 1998, 84, 6859.

[11] H. Kim, A. Pique, J. S. Horwitz, H. Mattoussi, H. Murata, Z. H. Kafafi, D. B. Chrisey, Appl. Phys. Lett. 1999, 74, 3444.

[12] K. Lin, R. S. Kumar, C. Peng, S. Lu, C. Soo-Jin, A. P. Burden, IEEE Photon. Technol. Lett. 2005, 17, 543.

[13] G. Gu, S. R. Forrest, IEEE J. Select. Top. Quantum Electron. 1998, 4, 83.

[14] V. Adamovich, A. Shoustikov, M. E. Thompson, Adv. Mater. 1999, $11,727$.

[15] H. Kim, C. M. Gilmore, J. S. Horwitz, A. Pique, H. Murata, G. P. Kushto, R. Schlaf, Z. H. Kafafi, D. B. Chrisey, Appl. Phys. Lett. 2000, 76, 259.

[16] A. Andersson, N. Johansson, P. Bröms, N. Yu, D. Lupo, W. R. Salaneck Adv. Mater. 1998, 10, 859 . 
[17] J. Cui, A. Wang, N. L. Edleman, J. Ni, P. Lee, N. R. Armstrong, T. J. Marks, Adv. Mater. 2001, 13, 1476.

[18] C. J. Lee, R. B. Pode, D. G. Moon, J. I. Han, Thin Solid Films 2004, 467, 201.

[19] C. F. Qiu, H. J. Peng, H. Y. Chen, Z. L. Xie, M. Wong, H. S. Kwok, IEEE Trans. Electron Devices 2004, 51, 1207.

[20] Z. Wu, S. Chen, H. Yang, Y. Zhao, J. Hou, S. Liu, Semicond. Sci. Technol. 2004, 19, 1138.

[21] H. J. Peng, X.- L. Zhu, J. X. Sun, Z. L. Xie, S. Xie, M. Wong, H.-S. Kwok, Appl. Phys. Lett. 2005, 87, 173505.

[22] Z. Wu, Z. Chen, X. Du, J. M. Logan, J. Sippel, M. Nikolou, K. Kamaras, J. R. Reynolds, D. B. Tanner, A. F. Hebard, A. G. Rinzler, Science 2004, 305, 1273.

[23] K. Lee, Z. Wu, Z. Chen, F. Ren, S. J. Pearton, A. G. Rinzler, Nano Lett. 2004, 4, 911.
[24] J. van de Lagemaat, T. M. Barnes, G. Rumbles, S. E. Shaheen, T. J. Coutts, C. Weeks, I. Levitsky, J. Peltola, P. Glatkowski, Appl. Phys. Lett. 2006, 88, 233503.

[25] M. W. Rowell, M. A. Topinka, M. D. McGehee, H.-J. Prall, G. Dennler, N. S. Sariciftci, L. Hu, G. Gruner, Appl. Phys. Lett. 2006, 88, 233506.

[26] S. Y. Chou, P. R. Krauss, P. J. Renstrom, Science 1996, $272,85$.

[27] L. J. Guo, Adv. Mater. 2007, 19, 495.

[28] J.-S. Kim, P. K. H. Ho, N. C. Greenham, R. H. Friend, J. Appl. Phys. 2000, 88, 1073.

[29] C. Liu, V. Kamaev, Z. V. Vardeny, Appl. Phys. Lett. 2005, 86, 143501.

[30] J. M. Ziebarth, A. K. Saafir, S. Fan, M. D. McGehee, Adv. Funct. Mater. 2004, 14, 451. 